$\xi=-1$

\title{
CoAP Monitoring System Using Logical Grouping Technique
}

\author{
JeongYun Kang, Nathali Silva, Kijun Han* \\ ${ }^{1}$ School of Computer Science and Engineering, Kyungpook National University, Daegu, Republic of Korea
}

\begin{abstract}
Most of IoT devices will have constraints in terms of memory add power. IETF proposed light-weight base Constrained Application Protocol (CoAP) at the application level. It supports two important features, observe and group communication. However, these two features are unable to work at the same time. In this paper, we propose a CoAP grouping technique using a gateway. Gateway generates device groups with resource type and saves groups' information in Database. It helps perform operations on the observed results and user can easily manage system.
\end{abstract}

Keywords: Internet of Things, CoAP, Constraind device, Wireless Sensor Network (WSN), Machine to Machine (M2M)

\section{Introduction}

Internet of things (IoT) is a concept which covers all kind of devices connected to each other, most of them being self-managed devices. These networks, in general, are composed of devices with limitations such as memory and power. IETF started targeting the efficient integration of constrained devices in web services and therefore established the Constrained RESTful Environments(CoRE) . CoRE's main goal is to standardize the Constrained Application Protocol (CoAP) protocol and replace HTTP on a Consolidated Device [1]. CoAP has Two important optional specifications, Group Communication [2] and Observe [3]. By those specifications, we can monitor CoAP resources or allowing interactions between multiple devices at once. However, these two features are unable to work at the same time [4]. Originally, CoAP only uses unicast, but in this paper proxy use broadcast to discover constrained devices at once.

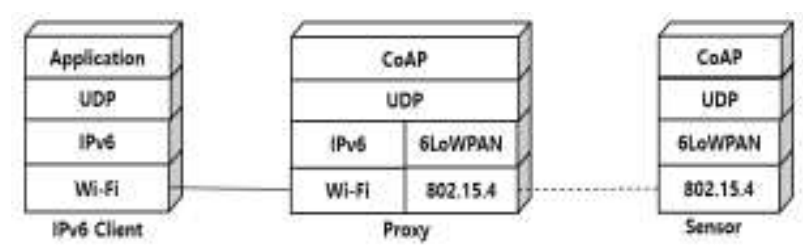

Figure 1:. IETF CoAP Protocol.

\section{Background}

CoAP is an application layer protocol that has been suggested by IETF. This protocol aims to enable constrained devices to run standard internet protocol. CoAP has been called RESTful application protocol. Also, HTTP is RESTful and CoAP becomes compatible with HTTP as shown in Fig. 1. By using a UDP, CoAP reduced overhead. through the 6LowPAN, it compressed IPv6 address. So, it helps IP used by Constrained device [5].

\section{IoT Monitoring by Logical CoAP Grouping}

This system consists of the CoAP Client, Proxy with Database, and numerous sensors. The element that manages the many sensors in the system is called Proxy. Proxy stores information of sensors in its database and sends control messages using information. The CoAP Client allows the user and proxy to interact and control each sensor. To do this, proxy must access the database and view resource information.

Client and Proxy are connected via Wi-Fi to send request messages remotely, on the other side Proxy and sensors are connected by BLE to reduce power consumption as shown in Fig. 2. As a first step in monitoring, when the CoAP Client sends a request message to Proxy, it sends a message to discover the CoAP server. Once sensors are found, the database divides them into groups according to the sensor resource type as Fig. 3 .

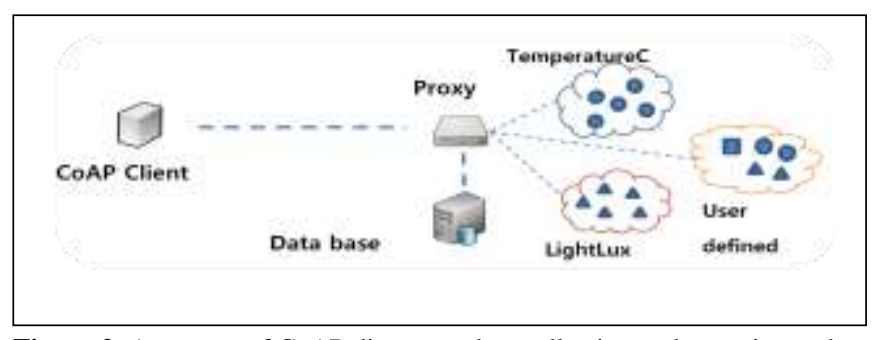

Figure 2. A process of CoAP discovery data collection and grouping technique

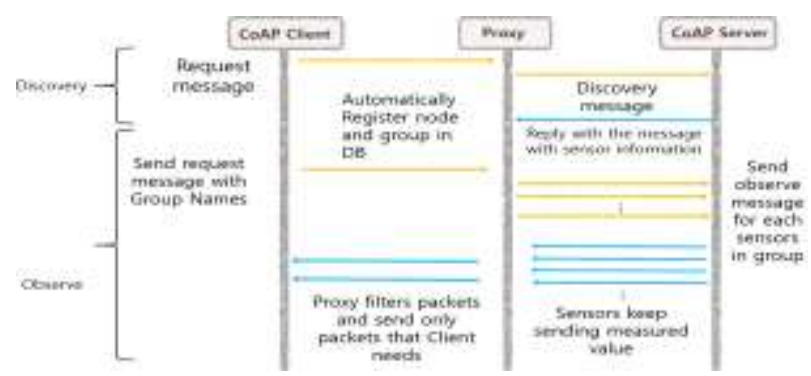

Figure 3:. Process of observe group 
This allows users to monitor a given environment by the type of data that they want and whether to return values for all resources. Grouped BLE sensors can be monitored by CoAP observe service. In order to monitor groups of sensors, the client registers observe with the group name. As mentioned afore, CoAP does not support broadcasting or multicasting with resources. Hence, if client sends request to CoAP Proxy, it sends observe registration message to BLE sensors with group interval in 1:1. CoAP server sends measurement data to proxy within the defined Interval time. Then, the proxy filters and sends data with respect to the threshold and priority. Consequently, it reduces proxy's burden.

\section{Performance Evaluation}

We compared discovery time of the conventional CoAP Wi-Fi sensors scenario with the proposed CoAP grouping technique. In standard case, proxy unicasts discovery message. Thus, as the number of CoAP Server increases, the number of packets received and sent increases too.

However, in proposed case, proxy sends only a request packet by broadcast. For that reason, in standard case, discovery time sharply increased. In the case of BLE Broadcast, if number of nodes increased, it causes retransmission. In other words, as the possibility of transmission increases the discovery time decreases. While BLE Unicast is not affected by the possibility of transmission. It was observed to see a significant decrease, going from 0 to 0.4 percent as you can see in Fig. 5 .

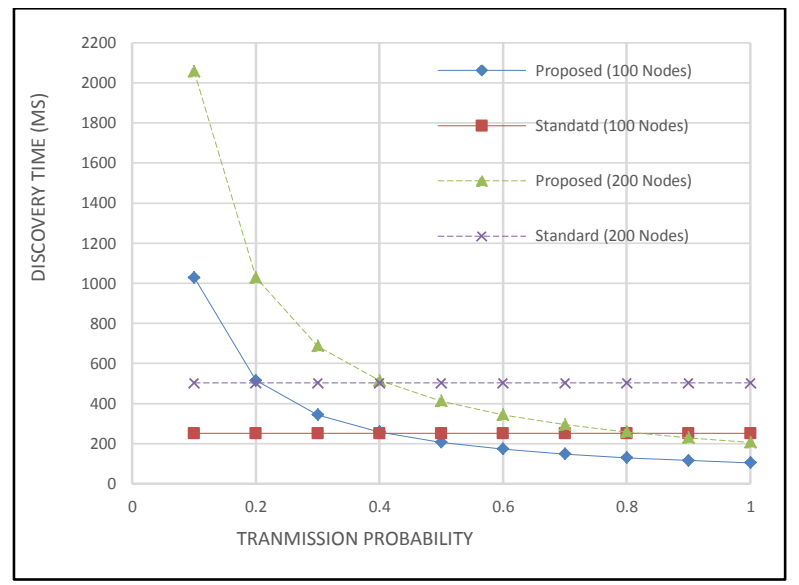

(a)

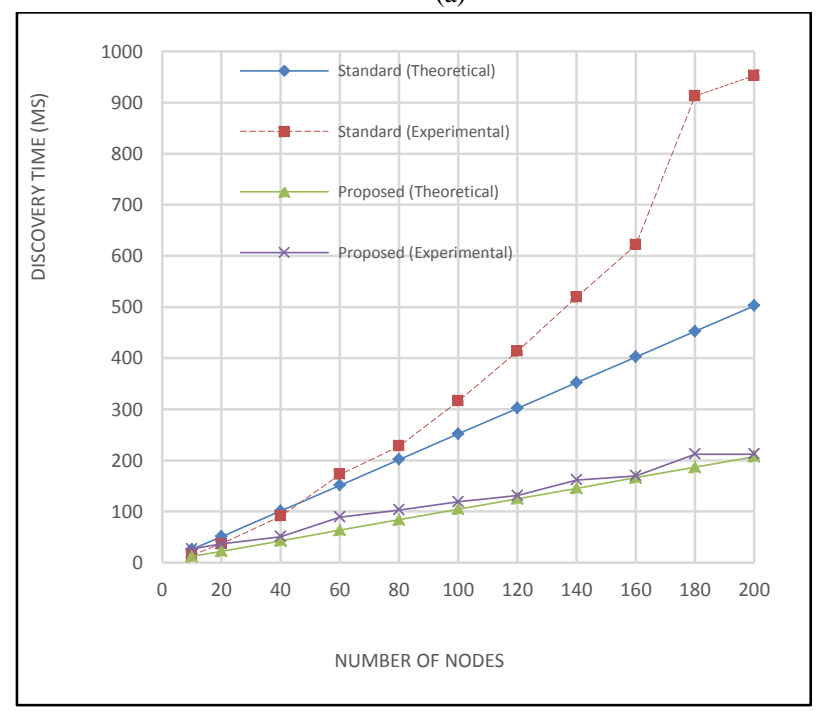

(b)

Figure 5:. Discovery time as the nodes are varied and discovery time as transmission probability increased.

\section{Conclusion}

Management of massive amount of IoT devices can be challenging due to the possibility of creating bottlenecks in the management server. In some scenarios, the server can even be a batteryoperated device or have a very narrow network bandwidth. In such scenarios, IoT traffic towards the server should be minimized, and possibly sent in a burst of traffic to minimize the energy consumption of the server. As a solution to the problems, we presented a proxy-based solution that can group devices together and an implementation of the proxy based on CoAP specifications. We evaluate its performance using JAVA codes. Depending on the number of sensors, the proposed scheme reduceed traffic to CoAP client by using a broadcast, priority, threshold. As future work, we intend to measure the power efficiency of the proxying approach using different wireless technologies

\section{Acknowledgment}

This work was supported by Institute for Information \& communications Technology Promotion (IITP) grant funded by the Korea government (MSIP) (No.2017-0-00770).

This study was supported by the BK21 Plus project (SW Human Resource Development Program for Supporting Smart Life) funded by the Ministry of Education, School of Computer Science and Engineering, Kyungpook National University, Korea (21A20131600005).

This research was supported by the Korean MSIT (Ministry of Science and ICT), under the National Program for Excellence in SW (2015-0-00912), supervised by the IITP (Institute for Information \& communications Technology Promotion)

\section{References}

[1] A. Rahman, E. Dijk, Group communication for the Constrained Application Protocol (CoAP), RFC, 7390, IETF, 2014.

[2] K. Hartke, observing resources in CoAP, Internet-Draft, draftietfcore-observe-16, IETF, 2014.

[3] K. Jeong-yun, J. Chang-Su, S. Ji-Hun, Y. Yong-Tak, H. Ki-Jun, A Multi-band Wearable Device System supporting Low-Energy Protocol, Proceedings of the Korean Society of Computer Information Con. 25 (2) (2017), 110-113.

[4] I. Ishaq, D. Carels, G.K. Teklemariam, J. Hoebeke, F.V.d. Abeele, E.D. Poorter, I.Moerman, P. Demeester, IETF standardization in the field of the Internet of things (iot): A survey, J. Sensor Actuator Netw. 2 (2) (2013), 235-287.

[5] Z. Shelby, K. Hartke, C. Bormann, The Constrained Application Protocol (CoAP), RFC, 7252, IETF, 2014. 